\title{
Misreported diagnosis of the Attention Deficit Hyperactivity Disorder
}

\author{
A. Giménez Palomo¹, A. Fernández-Fontelo², A. Cabaña ${ }^{3}$, D. Moriña ${ }^{3}$, P. Puig ${ }^{3}$.
}

${ }^{1}$ Department of Psychiatry. Institut Clínic de Neurociències. Hospital Clínic de Barcelona. Barcelona, Spain.

${ }^{2}$ Humboldt-Universität zu Berlin, Wirtschaftswissenschaftliche Fakultät, Berlin, Germany.

${ }^{3}$ Universitat Autònoma de Barcelona, Departament de Matemàtiques, Barcelona, Spain.

email address: agimenezp@clinic.cat

\section{INTRODUCTION}

Attention-deficit and hyperactivity disorder (ADHD) is one of the most common childhood and adolescent psychiatric disorders, with prevalence rates ranging from 5 to $7 \%$, and persisting into adulthood in approximately two-thirds of patients, being commonly underdiagnosed in this population. These symptoms create significant difficulties in academic attainment, executive functioning, and social interactions. To date, the diagnosis of ADHD mainly relies on clinical interviews, self/other reports, and rating scales. The inconsistency between the results of objective and subjective measurements has been reported in many studies on ADHD. In recent years, increasing attention is being paid to the development of objective evaluation tools. Many researchers in the area point out that it is strongly misdiagnosed since the criteria for diagnosis are still diffuse, and symptoms might dramatically differ depending on several characteristics (age, gender, etc.). This study presents a statistical model focused on the detection of misreporting of ADHD, being able to detect either over-reporting and under-reporting.

\section{OBJECTIVES}

Considering the statistic model based on the new operator, the following hypothesis about ADHD diagnosis are thoroughly studied: 1. Do the misreporting issue differ from males and females?; 2. Do the misreporting phenomenon vary between children, adolescents and adults?, and 3. Is the interaction between age and gender crucial to identify different misreporting patterns?

\section{METHODS}

The present work is aimed at quantifying the degree of misreporting or misdiagnosis in different settings of the official ADHD records in different regions of the province of Barcelona (Spain). In order to carry it out, the statistical models proposed by Fernández-Fontelo et al. (Under-reported data analysis with INAR-hidden Markov chains. Statistics in Medicine; 2016) and Fernández-Fontelo et al. (Untangling serially dependent under-reported count data from gender-based violence; under review) are extended by introducing a new operator, called fattening-thinning operator, which is able to assess both the phenomena of underreporting (or under-diagnosis) and over-reporting (or overdiagnosis).

\section{RESULTS}

Nearly 60 series among Barcelonès, Vallès Occidental and Baix Llobregat were included. Many of these areas, especially when boys and children were studied, showed clear annual and semiannual seasonal patterns (e.g., vacation periods).

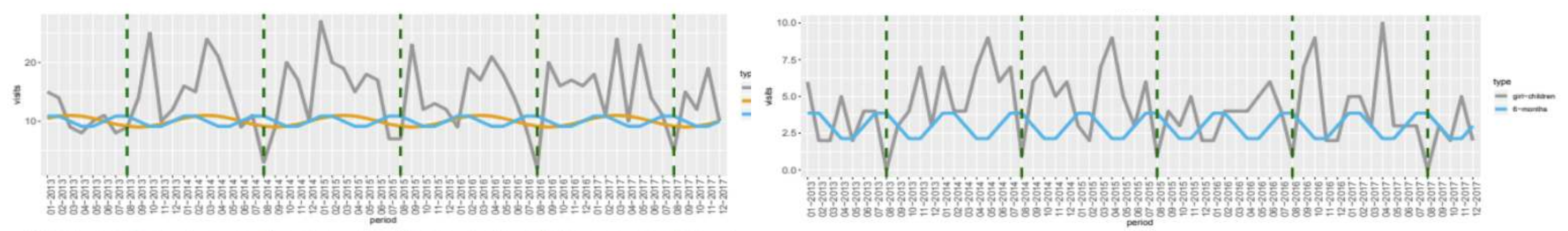

Seasonal patterns in boy-children and girl-children in Hospitalet de Llobregat, Barcelona.

\section{CONCLUSIONS}

This method should be conveniently validated in order to be applied in other populations, especially in mental health, where diagnostic tools are not commonly used and subjectivity plays an important role. Further discussions with clinicians are needed to confirm the differences in misreporting depending on geographical areas.

\section{REFERENCES}

